\title{
COMPACTNESS CONDITIONS IN UNIVERSAL ALGEBRAIC GEOMETRY
}

\author{
P. MODABBERI AND M. SHAHRYARI
}

\begin{abstract}
In this article, the properties of being equational noetherian, $q_{\omega}$ and $u_{\omega}$-compactness, and equational Artinian are studied from the perspective of the Zariski topology. The equational conditions on the relative free algebras of arbitrary varieties are also investigated and their relations to some logic and model theory notions are obtained. Some applications for the case of the universal algebraic geometry over groups are also introduced.
\end{abstract}

AMS Subject Classification Primary 03C99, Secondary 08A99 and 14 A99.

Keywords algebraic structures; equations; algebraic set; radical ideal; coordinate algebra; Zariski topology; noetherain algebra; equationally noetherian algebra; $q_{\omega}$-compactness; $u_{\omega}$-compactness; meta-noetherian algebras; meta-compact spaces; equational Artinian algebras; prevarieties; varieties; relative free algebras; chain conditions; eqautional domains; equational conditions; Hilbert's basis theorem.

\section{TABLE OF CONTENTS}

\section{INTRODUCTION}

\section{BASIC NOTIONS}

2.1 Systems of equations and algebraic sets

2.2 Radicals and coordinate algebras

2.3 Equational noetherian algebras

2.4 Unification theorems

\section{COMPACTNESS CONDITIONS}

3.1 Variants of equational conditions

$3.2 q_{\omega}$-compactness and Zariski topology

3.3 Meta-compact algebras

4. RELATIVELY FREE ALGEBRAS 
4.1 Relatively free algebras

4.2 Finitely axiomatizable classes

\section{SOME EQUATIONAL NOETHERIAN GROUPS}

\section{EQUATIONAL ARTINIAN ALGEBRAS}

\section{1 -radicals}

6.2 Relative systems of equations

6.3 Chain conditions on the ideals of relative free algebras

6.4 Hilbert's basis theorem

6.5 Examples of equational Artinian algebras

\section{OTHER TYPES OF EQUATIONAL CONDITIONS}

\section{REFERENCES}

\section{IntroduCtion}

Universal algebraic geometry is a new area of modern algebra, whose subject is basically the study of equations over an arbitrary algebraic structure $A$. In the classical algebraic geometry $A$ is a field. Many articles already published about algebraic geometry over groups, see [1], [2], 3], [4], and [5]. In an outstanding series of papers, O. Kharlampovich and A. Miyasnikov developed algebraic geometry over free groups to give affirmative answer for an old problem of Alfred Tarski concerning elementary theory of free groups (see [6] and also [7] for the independent solution of Z. Sela). Also in [8], a problem of Tarski about decidablity of the elementary theory of free groups is solved. Algebraic geometry over algebraic structures is also developed for algebras other than groups, for example there are results about algebraic geometry over Lie algebras and monoids, see [9], [10], and [11]. Systematic study of universal algebraic geometry is done in a series of articles by V. Remeslennikov, A. Myasnikov and E. Daniyarova in [12], [13], [14], and [15].

In this article, we are dealing with the equational conditions in the universal algebraic geometry, i.e. different conditions relating systems of equations especially conditions about systems and sub-systems of equations over algebras. The main examples of such conditions are equational noetherian property and its variants (weak equational noetherianity, $n$-equational noetherianity, $q_{\omega}$ and $u_{\omega}$-compactness), as well as equational Artinian property of algebras. We begin with a review of basic concepts of universal algebraic geometry and we describe the relation between the properties of being equational noetherian, $q_{\omega}$ 
and $u_{\omega}$-compactness and compactness of certain sets in the Zariski topology. We also discuss the concept of a meta-compact algebra and its relation to the meta-compactness of the sets in the Zariski topology. Then we introduce the notion of equational Artinian algebras. We provide some necessary conditions for a relative free algebra to be equational noetherian. We also show that the equational noetherianity of some relatively free algebras has interesting logical implications for certain subclasses of the corresponding variety. After defining the notion of relative systems of equations, we show that ascending (descending) chain conditions on the ideals of the relative free algebra is equivalent to equational noetherian (equational Artinian) property of all elements of the corresponding variety. A set of other types of interesting equational conditions and some questions is also presented at the end of the article.

\section{BASIC NOTIONS}

This section is devoted to a fast review of the basic concepts of the universal algebraic geometry. We suggest [16], [17] and [18] for reader who is not familiar to the universal algebra. The reader also would use [12], 13], 14], and [15], for extended exposition of the universal algebraic geometry. Our notations here are almost the same as in the above mentioned papers. Many results of this work can be stated for structures over any first order language, but for the sake of simplicity, we restrict ourself for the case of algebraic languages.

2.1. Systems of equations and algebraic sets. Suppose $\mathcal{L}$ is an arbitrary algebraic language and $A$ is a fixed algebra of type $\mathcal{L}$. The extended language will be denoted by $\mathcal{L}(A)$ and it is obtained from $\mathcal{L}$ by adding new constant symbols $a \in A$. An algebra $B$ of type $\mathcal{L}(A)$ is called $A$-algebra, if the map $a \mapsto a^{B}$ is an embedding of $A$ in $B$. Note that here, $a^{B}$ denotes the interpretation of the constant symbol $a$ in $B$. We assume that $X=\left\{x_{1}, \ldots, x_{n}\right\}$ is a finite set of variables. We denote the term algebra in the language $\mathcal{L}$ and variables from $X$ by $T_{\mathcal{L}}(X)$, and similarly the term algebra in the extended language $\mathcal{L}(A)$ will denoted by $T_{\mathcal{L}(A)}(X)$. For the sake of simplicity, we define our notions in the coefficient free frame, i.e. in the language $\mathcal{L}$ and then we can extend all the definitions to the language $\mathcal{L}(A)$.

Fix an algebraic language $\mathcal{L}$ and a set of variables $X=\left\{x_{1}, \ldots, x_{n}\right\}$. An equation is a pair $(p, q)$ of the elements of the term algebra $T_{\mathcal{L}}(X)$. In many cases, we assume that such an equation is the same as the atomic formula $p\left(x_{1}, \ldots, x_{n}\right) \approx q\left(x_{1}, \ldots, x_{n}\right)$ or $p \approx q$ in short. Hence, 
in this article the set $A t_{\mathcal{L}}(X)$ of atomic formulae in the language $\mathcal{L}$ and the product algebra $T_{\mathcal{L}}(X) \times T_{\mathcal{L}}(X)$ are assumed to be equal.

Any subset $S \subseteq A t_{\mathcal{L}}(X)$ is called a system of equations in the language $\mathcal{L}$. A system $S$ is called consistent over an algebra $A$, if there is an element $\left(a_{1}, \ldots, a_{n}\right) \in A^{n}$ such that for all equations $(p \approx q) \in S$, the equality

$$
p^{A}\left(a_{1}, \ldots, a_{n}\right)=q^{A}\left(a_{1}, \ldots, a_{n}\right)
$$

holds. Otherwise, we say that $S$ is in-consistent over $A$. Note that, $p^{A}$ and $q^{A}$ are the corresponding term functions on $A^{n}$. A system of equations $S$ is called an ideal, if it corresponds to a congruence on $T_{\mathcal{L}}(X)$. For an arbitrary system of equations $S$, the ideal generated by $S$, is the smallest congruence containing $S$ and it is denoted by $[S]$.

For an algebra $A$ of type $\mathcal{L}$, an element $\left(a_{1}, \ldots, a_{n}\right) \in A^{n}$ will be denoted by $\bar{a}$, sometimes. Let $S$ be a system of equations. Then the set

$$
V_{A}(S)=\left\{\bar{a} \in A^{n}: \forall(p \approx q) \in S, p^{A}(\bar{a})=q^{A}(\bar{a})\right\}
$$

is called an algebraic set. It is clear that for any non-empty family $\left\{S_{i}\right\}_{i \in I}$, we have

$$
V_{A}\left(\bigcup_{i \in I} S_{i}\right)=\bigcap_{i \in I} V_{A}\left(S_{i}\right)
$$

So, we define a closed set in $A^{n}$ to be an arbitrary intersections of finite unions of algebraic sets. Therefore, we obtain a topology on $A^{n}$, which is called Zariski topology. For a subset $Y \subseteq A^{n}$, its closure with respect to Zariski topology is denoted by $\bar{Y}$. We also denote by $Y^{a c}$ the smallest algebraic set containing $Y$. In general $\bar{Y}=Y^{a c}$ is not true. We say that an algebra $A$ is equational domain, if the union of two algebraic set in $A^{n}$ is again an algebraic set for any $n$. In this case, clearly we have the equality $\bar{Y}=Y^{a c}$. In the next section, we will prove some of our results in the case of equational domains, therefore we should mention here that there is a long list of known examples of such algebras. For details, the reader can see [15], where there are also some interesting criteria for an algebra to be equational domain.

Similarly, we can work with systems of equations with coefficients from a fixed algebra $A$. To do this, let $A$ be an algebra of type $\mathcal{L}$ and consider a pair of terms $(p, q)$ in the extended language $\mathcal{L}(A)$. Then we call the atomic formula $p \approx q$ an equation with coefficient from $A$. We can define a system of equations with coefficients from $A$, so for any such system $S$ and any $A$-algebra $B$, we can define the algebraic set $V_{B}(S)$. Other notions may also be defined in a similar manner. Note that some examples of equational domain appear in the recent case, for example, as it is proved in [15], no non-trivial group is equational 
domain in the language $\mathcal{L}=\left(1,{ }^{-1}, \cdot\right)$ of groups, but any non-abelian free group $F$ is equational domain in the language $\mathcal{L}(F)$.

Almost all results of the next section may also stated for the general case of $A$-algebras and systems in the extended language $\mathcal{L}(A)$, but for the sake of simplicity, we restrict ourself to the coefficient free case.

2.2. Radicals and coordinate algebras. For any set $Y \subseteq A^{n}$, we define

$$
\operatorname{Rad}(Y)=\left\{(p, q): \forall \bar{a} \in Y, p^{A}(\bar{a})=q^{A}(\bar{a})\right\} .
$$

It is easy to see that $\operatorname{Rad}(Y)$ is an ideal in the term algebra. Any ideal of this type is called an A-radical ideal or a radical ideal for short. Note that any ideal in the term algebra is in fact a radical ideal. To see the reason, just note that for any ideal $R$ in the term algebra $T_{\mathcal{L}}(X)$, if we consider the algebra $B(R)=T_{\mathcal{L}}(X) / R$, then $\operatorname{Rad}_{B(R)}(R)=R$.

It is easy to see that a set $Y$ is algebraic if and only if $V_{A}(\operatorname{Rad}(Y))=$ $Y$. In the general case, we have $V_{A}(\operatorname{Rad}(Y))=Y^{a c}$, see [13]. The coordinate algebra of a set $Y$ is the quotient algebra

$$
\Gamma(Y)=\frac{T_{\mathcal{L}}(X)}{\operatorname{Rad}(Y)}
$$

An arbitrary element of $\Gamma(Y)$ is denoted by $[p]_{Y}$. We define a function $p^{Y}: Y \rightarrow A$ by the rule

$$
p^{Y}(\bar{a})=p^{A}\left(a_{1}, \ldots, a_{n}\right),
$$

which is a term function on $Y$. The set of all such functions will be denoted by $T(Y)$ and it is naturally an algebra of type $\mathcal{L}$. It is easy to see that the map $[p]_{Y} \mapsto p^{Y}$ is a well-defined isomorphism. So, we have $\Gamma(Y) \cong T(Y)$.

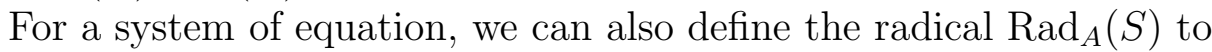
be $\operatorname{Rad}\left(V_{A}(S)\right)$. Two systems $S$ and $S^{\prime}$ are called equivalent over $A$, if they have the same set of solutions in $A$, i.e. $V_{A}(S)=V_{A}\left(S^{\prime}\right)$. So, clearly $\operatorname{Rad}_{A}(S)$ is the largest system which is equivalent to $S$. Note that $[S] \subseteq \operatorname{Rad}_{A}(S)$.

One of the major problems of the universal algebraic geometry is to determine the structures of algebras which appear as the coordinate algebras. There are many necessary and sufficient conditions for an algebra to be a coordinate algebra and we will give a summary of such results in the subsection 2.4 .

2.3. Equational noetherian algebras. In this article, we are dealing with equational conditions on algebras. The first and maybe the most important condition of this type can be formulated as follows. 
Definition 1. An algebra $A$ is called equational noetherian, if for any system of equations $S$, there exists a finite subsystem $S_{0} \subseteq S$, which is equivalent to $S$ over $A$, i.e. $V_{A}(S)=V_{A}\left(S_{0}\right)$.

Note that we can consider a bound on the number of variables appearing in $S$ and obtain a weaker notion of $n$-equational noetherian algebra; an algebra $A$ is $n$-equational noetherian, if for any system $S$ with at most $n$ variables, there exists a subsystem $S_{0} \subseteq S$, which is equivalent to $S$ over $A$, i.e. $V_{A}(S)=V_{A}\left(S_{0}\right)$.

If an $A$-algebra is equational noetherian in the language $\mathcal{L}(A)$, then we call it $A$-equational noetherian. Many examples of equational noetherian algebras are introduced in [13. Among them are noetherian rings and linear groups over noetherian rings as well as free groups. In [13], it is proved that the next four assertions are equivalent:

$i$ - An algebra $A$ is equational noetherian.

ii- For any system $S$, there exists a finite $S_{0} \subseteq[S]$, such that $V_{A}(S)=$ $V_{A}\left(S_{0}\right)$.

iii- For any $n$, the Zariski topology on $A^{n}$ is noetherian, i.e. any descending chain of closed subsets terminates.

iv- Any chain of coordinate algebras and epimorphisims

$$
\Gamma\left(Y_{1}\right) \rightarrow \Gamma\left(Y_{2}\right) \rightarrow \Gamma\left(Y_{3}\right) \rightarrow \cdots
$$

terminates.

So, in the case of equational noetherian algebras, any closed set in $A^{n}$ is equal to a minimal finite union of irreducible algebraic sets which is unique up to a permutation. Note that a set is called irreducible, if it has no proper finite covering consisting of closed sets. The following theorem is proved in [13].

Theorem 1. Let $A$ be an equational noetherian algebra. Then the following algebras are also equational noetherian:

$i$ - any subalgebra and filter-power of $A$.

ii- any coordinate algebra over $A$.

iii- any fully residually A-algebra. 
$i v$ - any algebra belonging to the quasi-variety generated by $A$.

$v$ - any algebra universally equivalent to $A$.

vi- any limit algebra over $A$.

vii- any finitely generated algebra defined by a complete atomic type in the universal theory of $A$ or in the set of quasi-identities of $A$.

We can generalize the concept of equational noetherian algebras by dropping the condition $S_{0} \subseteq S$ in the definition 1. More precisely we have;

Definition 2. An algebra $A$ is weak equational noetherian, if for any system $S$ there exists a finite system $S_{0}$, equivalent to $S$ over $A$.

Equivalently, an algebra $A$ is weak equational noetherian, if and only if for any system $S$, the radical ideal $\operatorname{Rad}_{A}(S)$ is finitely generated, i.e. there exists a finite $S_{0} \subseteq \operatorname{Rad}_{A}(S)$ such that $\operatorname{Rad}_{A}(S)=\operatorname{Rad}_{A}\left(S_{0}\right)$. We can explain the logical meaning of this equality as follows. Let $\mathrm{QId}(A)$ be the set of quasi-identities of $A$. Then $A$ is weak equational noetherian iff, for any system of equations $S$, exist finitely many equation $p_{1} \approx q_{1}, \ldots, p_{m} \approx q_{m}$ such that

$$
\operatorname{Rad}_{A}(S)=\left\{(p, q):\left(\forall x_{1} \ldots \forall x_{n}\left(\bigwedge_{i=1}^{m} p_{i} \approx q_{i} \Rightarrow p \approx q\right)\right) \in \operatorname{QId}(A)\right\} .
$$

2.4. Unification theorems. Many variants of the unification theorems are proved for universal algebraic geometry in [12], [13] and [14]. The main aim of this type of theorems is to determine algebras which are the coordinate algebra of an algebraic set. In this subsection, we discuss just one of these unification theorems and the reader can consult the above mentioned articles for detailed exposition of notions and proofs.

Theorem 2. Let $A$ and $\Gamma$ be algebras in a language $\mathcal{L}$. Suppose $A$ is equational noetherian and $\Gamma$ is finitely generated. Then the following assertions are equivalent.

$i$ - $\Gamma$ is the coordinate algebra of some irreducible algebraic set over $A$.

ii- $\Gamma$ is a fully residually A-algebra. This means that for any finite subset $C \subseteq \Gamma$, there exists a homomorphism $\alpha: \Gamma \rightarrow A$, such that the restriction of $\alpha$ to $C$ is injective. 
iii- $\Gamma$ embeds into some ultra-power of $A$.

$i v$ - $\Gamma$ belongs to the universal closure of $A$, i.e. $T h_{\forall}(A) \subseteq T h_{\forall}(\Gamma)$.

$v$ - $\Gamma$ is a limit algebra over $A$.

vi- $\Gamma$ is defined by a complete type in $T h_{\forall}(A)$.

There are similar theorems for the cases where $A$ is weak equational noetherian, or it is $q_{\omega}$-compact or $u_{\omega}$-compact. See [14] for a detailed discussion.

\section{Compactness Conditions}

In this section we study some of the important equational conditions over algebras by means of the Zariski topology. The notions of $q_{\omega}$ and $u_{\omega}$-compact algebras are introduced in [14. In the equational domain case, we will give a topological characterization of $q_{\omega}$ and $u_{\omega}$-compact algebras. We also introduce a new class of algebras which generalizes the class of equational noetherian algebras using the concept of metacompact topological spaces.

3.1. Variants of equational conditions. Recall that an algebra $A$ is equational noetherian if and only if any system of equations is equivalent to a finite subsystem over $A$. We also defined weak equational noetherian algebras in the previous section. Both of these properties are defined by applying certain conditions on the systems of equations. Clearly, one can use many different conditions to obtain new classes of algebras. Here we introduce two more examples of such classes.

Definition 3. An algebra $A$ is called $q_{\omega}$-compact if for any system of equations $S$ and any equation $p \approx q$ with $V_{A}(S) \subseteq V_{A}(p \approx q)$, there exists a finite subset $S_{0} \subseteq S$ such that $V_{A}\left(S_{0}\right) \subseteq V_{A}(p \approx q)$. Similarly $A$ is called $u_{\omega}$-compact, if for any arbitrary system $S$ and any finite system $\left\{p_{1} \approx q_{1}, \ldots, p_{m} \approx q_{m}\right\}$, the inclusion

$$
V_{A}(S) \subseteq \bigcup_{i=1}^{m} V_{A}\left(p_{i} \approx q_{i}\right)
$$

implies the existence of a finite subset $S_{0} \subseteq S$ such that

$$
V_{A}\left(S_{0}\right) \subseteq \bigcup_{i=1}^{m} V_{A}\left(p_{i} \approx q_{i}\right)
$$


It is easy to see that any equational noetherian algebra is $u_{\omega}$-compact and any $u_{\omega}$-compact algebra is $q_{\omega}$-compact. The converse statements are not true and the reader may see [14] for some counterexamples. The unification theorem is proved for the algebras in these new classes, [14. As in the case of $n$-equational noetherian algebras (2.3), we can also define $q_{\omega}^{n}$-compact algebras as well as $u_{\omega}^{n}$-compact algebras.

In the next subsection, we give topological characterization for $q_{\omega}$ and $u_{\omega}$-compact algebras. Note that, an algebra $A$ is equational noetherian if and only if for all $n$, the space $A^{n}$ is noetherian and this is equivalent to say that any subset of $A^{n}$ is compact in the Zariski topology. To see this, suppose for example every subset of $A^{n}$ is compact and $S$ is an arbitrary system of equations. Clearly we have

$$
V_{A}(S)=\bigcap_{(p \approx q) \in S} V_{A}(p \approx q),
$$

hence equivalently

$$
A^{n} \backslash V_{A}(S)=\bigcup_{(p \approx q) \in S} A^{n} \backslash V_{A}(p \approx q) .
$$

By the compactness, there are finite number of equations $p_{1} \approx q_{1}, \ldots, p_{m} \approx$ $q_{m}$ in $S$, such that

$$
A^{n} \backslash V_{A}(S)=\bigcup_{i=1}^{m} A^{n} \backslash V_{A}\left(p_{i} \approx q_{i}\right) .
$$

This shows that $V_{A}(S)=V_{A}\left(S_{0}\right)$, where $S_{0}=\left\{p_{1} \approx q_{1}, \ldots, p_{m} \approx q_{m}\right\}$. Therefore $A$ is equational noetherian. It can be easily shown that the converse is also true. So we have

Proposition 1. An algebra $A$ is equational noetherian if and only if, for any $n$ all subsets of $A^{n}$ are compact.

This proposition is our main motivation to investigate similar criteria for the case of $q_{\omega}$ and $u_{\omega}$-compact algebras.

3.2. $q_{\omega}$-compactness and Zariski topology. Let $A$ be an algebra in a language $\mathcal{L}$ and $p \approx q$ be an equation. We denote the open set $A^{n} \backslash V_{A}(p \approx q)$ by $C_{A}(p \approx q)$.

Proposition 2. Let $A$ be an equational domain. Then $A$ is $q_{\omega}$-compact if and only if $C_{A}(p \approx q)$ is compact for all $p \approx q$.

Proof. First suppose $A$ is $q_{\omega}$-compact. Let $C_{A}(p \approx q) \subseteq \bigcup_{i \in I} C_{i}$, with $C_{i} \subseteq A^{n}$ open. Since $A$ is equational domain, so $C_{i}=A^{n} \backslash V_{A}\left(S_{i}\right)$ for 
some system $S_{i}$. We have

$$
A^{n} \backslash V_{A}(p \approx q) \subseteq \bigcup_{i \in I}\left(A^{n} \backslash V_{A}\left(S_{i}\right)\right)=A^{n} \backslash \bigcap_{i \in I} V_{A}\left(S_{i}\right)
$$

hence

$$
\bigcap_{i \in I} V_{A}\left(S_{i}\right) \subseteq V_{A}(p \approx q)
$$

This shows that

$$
V_{A}\left(\bigcup_{i \in I} S_{i}\right) \subseteq V_{A}(p \approx q)
$$

and so, there is a finite $S^{\prime} \subseteq \bigcup_{i \in I} S_{i}$, with $V_{A}\left(S^{\prime}\right) \subseteq V_{A}(p \approx q)$. We have

$$
S^{\prime} \subseteq S_{i_{1}} \cup \cdots \cup S_{i_{m}}
$$

for some $i_{1}, \ldots, i_{m} \in I$. Hence

$$
\bigcap_{j=1}^{m} V_{A}\left(S_{i_{j}}\right) \subseteq V_{A}\left(S^{\prime}\right) \subseteq V_{A}(p \approx q),
$$

and therefore $C_{A}(p \approx q) \subseteq \bigcup_{j=1}^{m} C_{i_{j}}$. This shows that $C_{A}(p \approx q)$ is compact. Conversely, suppose any $C_{A}(p \approx q)$ is compact. Let $V_{A}(S) \subseteq$ $V_{A}(p \approx q)$. Then

$$
\begin{aligned}
C_{A}(p \approx q) & \subseteq A^{n} \backslash V_{A}(S) \\
& =A^{n} \backslash \bigcap_{(p \approx q) \in S} V_{A}(p \approx q) \\
& =\bigcup_{(p \approx q) \in S}\left(A^{n} \backslash V_{A}(p \approx q)\right) .
\end{aligned}
$$

Hence

$$
C_{A}(p \approx q) \subseteq \bigcup_{i=1}^{m}\left(A^{n} \backslash V_{A}\left(p_{i} \approx q_{i}\right)\right),
$$

with $p_{1} \approx q_{1}, \ldots, p_{m} \approx q_{m} \in S$. Therefore

$$
V_{A}\left(p_{1} \approx q_{1}, \ldots, p_{m} \approx q_{m}\right) \subseteq V_{A}(p \approx q),
$$

and so, $A$ is $q_{\omega}$-compact.

A similar result is true for $u_{\omega}$-compact equational domains. It can be shown that an equational domain $A$ is $u_{\omega}$-compact if and only if any finite intersection of sets of the form $C_{A}(p \approx q)$ is compact. 
3.3. Meta-compact algebras. A topological space is called metacompact if every open covering of it, has a refinement which is also a covering and every point belongs to finitely many element of the refinement. Motivating by meta-compact topological spaces, we define meta-compact algebras. Let $S$ be a system of equations in a language $\mathcal{L}$ and $A$ be an algebra. We denote by $V_{A}^{*}(S)$ the set of all points $\left(a_{1}, \ldots, a_{n}\right) \in A^{n}$ such that for all but finitely many equations $(p \approx$ $q) \in S$, we have $p^{A}\left(a_{1}, \ldots, a_{n}\right)=q^{A}\left(a_{1}, \ldots, a_{n}\right)$.

Definition 4. Let for any in-consistent system $S$ over $A$, there exists an in-consistent subsystem $S^{\prime} \subseteq S$, such that $V_{A}^{*}\left(S^{\prime}\right)=A^{n}$. Then we call $A$ a meta-compact algebra.

Any equational noetherian algebra is also meta-compact. This is because, if $A$ is equational noetherian and $S$ is an in-consistent system, then there is a finite $S_{o} \subseteq S$ such that $V_{A}\left(S_{0}\right)=V_{A}(S)$ and so, $S_{0}$ is also in-consistent. But since $S_{0}$ is finite, so we have clearly $V_{A}^{*}\left(S_{0}\right)=A^{n}$. Hence, $A$ is meta-compact.

Proposition 3. Let $A$ be meta-compact equational domain. Then for any $n$ the space $A^{n}$ is a meta-compact topological space.

Proof. Let $A^{n}=\bigcup_{\alpha \in I} C_{\alpha}$ be a covering of $A^{n}$, indexed by a set of ordinals $I=\{\alpha: \alpha \leq \kappa\}$. Since $A$ is equational domain, every $C_{\alpha}$ has the form

$$
C_{\alpha}=A^{n} \backslash V_{A}\left(S_{\alpha}\right)
$$

for some system of equations $S_{\alpha}$. We have $\bigcap_{\alpha} V_{A}\left(S_{\alpha}\right)=\emptyset$. Suppose $S=\bigcup_{\alpha} S_{\alpha}$. Then $V_{A}(S)=\emptyset$, and therefore there exists an in-consistent subsystem $S^{\prime}$, such that $V_{A}^{*}\left(S^{\prime}\right)=A^{n}$. Define by transfinite induction

$$
\begin{aligned}
S_{0}^{\prime} & =S^{\prime} \cap S_{0}, \\
S_{\alpha^{+}}^{\prime} & =\left(S^{\prime} \cap S_{\alpha^{+}}\right) \backslash S_{\alpha}^{\prime},
\end{aligned}
$$

and for any limit ordinal, we set

$$
S_{\lambda}^{\prime}=\left(S^{\prime} \cap S_{\lambda}\right) \backslash \bigcup_{\alpha<\lambda} S_{\alpha}^{\prime} .
$$

We have clearly $S^{\prime}=\bigcup_{\alpha} S_{\alpha}^{\prime}, S_{\alpha}^{\prime} \subseteq S_{\alpha}$, and $S_{\alpha}^{\prime} \cap S_{\beta}^{\prime}=\emptyset$, for any distinct $\alpha$ and $\beta$. Now, let

$$
C_{\alpha}^{\prime}=A^{n} \backslash V_{A}\left(S_{\alpha}^{\prime}\right)
$$

We have $C_{\alpha}^{\prime} \subseteq C_{\alpha}$ and $\bigcup_{\alpha} C_{\alpha}^{\prime}=A^{n}$. Hence, we obtain a refinement of the given covering. Now, for $\bar{a} \in A^{n}$, we have $\bar{a} \in V_{A}^{*}\left(S^{\prime}\right)$, so there are finitely many equations

$$
p_{1} \approx q_{1}, \ldots, p_{m} \approx q_{m} \in S^{\prime}
$$


such that $p_{i}^{A}(\bar{a}) \neq q_{i}^{A}(\bar{a})$, for $1 \leq i \leq m$. For any $i$, there exists a unique $\alpha_{i} \in I$ such that $\left(p_{i} \approx q_{i}\right) \in S_{\alpha_{i}}^{\prime}$. Therefore, $\bar{a}$ does not belong to

$$
V_{A}\left(S_{\alpha_{1}}^{\prime}\right), \ldots, V_{A}\left(S_{\alpha_{m}}^{\prime}\right)
$$

and for other $\alpha \in I$, we have $\bar{a} \in V_{A}\left(S_{\alpha}^{\prime}\right)$ (since $S_{\alpha_{i}}^{\prime} \cap S_{\alpha}^{\prime}=\emptyset$ ). This shows that $\bar{a}$ just belongs to $C_{\alpha_{1}}^{\prime}, \ldots, C_{\alpha_{m}}^{\prime}$. We now, proved that $A^{n}$ is meta-compact.

\section{Relatively fReE Algebras}

In this section, we study the universal algebraic geometry of the relatively free algebras. We show that for a variety $\mathbf{V}$, any solution of an equation over the relatively free algebras of $\mathbf{V}$ is corresponds to an identity in $\mathbf{V}$. This idea, leads us to obtain some interesting results concerning classes of algebras (especially varieties) using concepts of the universal algebraic geometry.

4.1. Relatively free algebras. One of the major tools in the universal algebraic geometry is the relatively free algebra of a given variety over a given set of variables. We can discuss this notion in more general framework of pre-varieties. A class $\mathbf{V}$ of algebras of type $\mathcal{L}$ is a pre-variety, if it is closed under the operations of taking subalgebra and arbitrary direct product. For an arbitrary algebra $A$, we denote the set of all congruences of $A$ by $\operatorname{Cong}(A)$. If $\mathbf{V}$ is a pre-variety and $X$ is a set of variables, we can define an ideal of the term algebra $T_{\mathcal{L}}(X)$ by

$$
R_{\mathbf{V}}(X)=\bigcap\left\{R \in \operatorname{Cong}\left(T_{\mathcal{L}}(X)\right): \frac{T_{\mathcal{L}}(X)}{R} \in \mathbf{V}\right\} .
$$

So, $R_{\mathbf{V}}(X)$ is the smallest congruence in the term algebra such that the corresponding quotient belongs to $\mathbf{V}$. The quotient algebra

$$
F_{\mathbf{V}}(X)=\frac{T_{\mathcal{L}}(X)}{R_{\mathbf{V}}(X)}
$$

is called the relative free algebra over $X$ in $\mathbf{V}$. It is a member of $\mathbf{V}$ and it can be characterized by the universal mapping property: it is generated by the set $\bar{X}=\left\{x / R_{\mathbf{V}}(X): x \in X\right\}$ and any map from $\bar{X}$ to an algebra $A \in \mathbf{V}$ extends uniquely to a homomorphism from $F_{\mathbf{V}}(X)$ to $A$. It can be easily seen that $|X|=|\bar{X}|$. More details on the universal mapping property can be find in [16]. We also have a logical characterization of $R_{\mathbf{V}}(X)$.

Lemma 1. Let $\mathbf{V}$ be a pre-variety and $X$ be a set of variables. Then

$$
R_{\mathbf{V}}(X)=\left\{(p, q): V \vDash\left(\forall x_{1} \ldots \forall x_{n} p \approx q\right)\right\} .
$$


Proof. We prove the assertion for finite $X$ and by a small modification, it can be proved for infinite set of variables. Note that if $p$ and $q$ are terms with variables $x_{1}, \ldots, x_{n}$, then $\mathbf{V} \vDash\left(\forall x_{1} \ldots \forall x_{n} p \approx q\right)$ means that for all $A \in \mathbf{V}$ and all $a_{1}, \ldots, a_{n} \in A$, we have the equality

$$
p^{A}\left(a_{1}, \ldots, a_{n}\right)=q^{A}\left(a_{1}, \ldots, a_{n}\right) .
$$

To prove the lemma, assume that

$$
K=\left\{(p, q): V \vDash\left(\forall x_{1} \ldots \forall x_{n} p \approx q\right)\right\} .
$$

Let $R$ be an ideal in $T_{\mathcal{L}}(X)$ such that $T_{\mathcal{L}}(X) / R \in \mathbf{V}$ and let $(p, q) \in K$. If we let $A=T_{\mathcal{L}}(X) / R$, then

$$
p^{A}\left(x_{1} / R, \ldots, x_{n} / R\right)=q^{A}\left(x_{1} / R, \ldots, x_{n} / R\right),
$$

where $x / R$ denotes the class containing $x$. This equality is equivalent to $p / R=q / R$, so $(p, q) \in R$. This proves that $K \subseteq R_{\mathbf{V}}$.

To see the inverse inclusion, let $F=T_{\mathcal{L}}(X) / K$, which is generated by the set $X^{*}=\{x / K: x \in X\}$. We show that $F$ has the universal mapping property with respect to the set $X^{*}$ and the pre-variety $\mathbf{V}$. Let $\alpha: X^{*} \rightarrow A$ be any map, where $A \in \mathbf{V}$. Define $\alpha_{0}: X \rightarrow A$ by $\alpha_{0}(x)=\alpha(x / K)$. We know that there exists a homomorphism $\alpha_{0}^{\prime}: T_{\mathcal{L}}(X) \rightarrow A$, extending $\alpha_{0}$. It is easy to see that for all term $p \in T_{\mathcal{L}}(X)$, we have $\alpha_{0}^{\prime}(p)=p^{A}\left(\alpha\left(x_{1} / K\right), \ldots, \alpha\left(x_{n} / K\right)\right)$. This shows that for $(p, q) \in K$, we have $\alpha_{0}^{\prime}(p)=\alpha_{0}^{\prime}(q)$, and hence $(p, q) \in \operatorname{ker} \alpha_{0}^{\prime}$. Therefore we have a homomorphism $\alpha^{\prime}: F \rightarrow A$ such that

$$
\alpha^{\prime}(t / K)=\alpha_{0}^{\prime}(t) \text {. }
$$

Clearly, $\alpha^{\prime}$ coincides with $\alpha$ over $X^{*}$. We show that $\alpha^{\prime}$ is unique. Let $h: F \rightarrow A$ be another homomorphism such that $h$ coincides with $\alpha$ over $X^{*}$. Using induction on the complexity of the term $t=f\left(t_{1}, \ldots, t_{m}\right)$, we have

$$
\begin{aligned}
h(t / K) & =h\left(\frac{f\left(t_{1}, \ldots, t_{m}\right)}{K}\right) \\
& =f^{A}\left(h\left(t_{1} / K\right), \ldots, h\left(t_{m} / K\right)\right) \\
& =f^{A}\left(\alpha^{\prime}\left(t_{1} / K\right), \ldots, \alpha^{\prime}\left(t_{m} / K\right)\right) \\
& =\alpha^{\prime}\left(\frac{f\left(t_{1}, \ldots, t_{m}\right)}{K}\right) \\
& =\alpha^{\prime}(t / K) .
\end{aligned}
$$

This argument shows that $F$ is free relative to $\mathbf{V}$ and hence it belongs to $\mathbf{V}$. Therefore $R_{\mathbf{V}}(X) \subseteq K$.

We give another interpretation of this lemma using the terminology of the equational logic of Tarski. Recall that a congruence of the term 
algebra $T_{\mathcal{L}}\left(x_{1}, x_{2}, \ldots\right)$ is called fully invariant, if it is invariant under any endomorphism of the term algebra. For any set $\Sigma$ of identities, $\Theta_{f i}(\Sigma)$ denotes the fully invariant closure of $\Sigma$. As in [16], this set is equal to the deductive closure of $\Sigma$, i.e.

$$
\Theta_{f i}(\Sigma)=D(\Sigma)
$$

Now, let $\mathbf{V}$ be a variety of algebras in the language $\mathcal{L}$. Let $\Sigma$ be a set of identities for $\mathbf{V}$ with variables from $X$. Then the above lemmas says that $R_{\mathbf{V}}(X)=D(\Sigma)$. The next result will be used in the subsequence parts of this article.

Corollary 1. Let $\mathbf{V}$ be a pre-variety of algebras in a language $\mathcal{L}$ and $X$ be a set. Let $F=F_{\mathbf{V}}(X)$ and $p \approx q$ be an equation with $n$ indeterminate. Then $\left(\bar{t}_{1}, \ldots, \bar{t}_{n}\right) \in F^{n}$ is a solution of $p \approx q$, if and only if

$$
\forall x_{1} \ldots \forall x_{m} p\left(t_{1}, \ldots, t_{n}\right) \approx q\left(t_{1}, \ldots, t_{n}\right)
$$

is an identity in $\mathbf{V}$. Here $x_{1}, \ldots, x_{m}$ are the variables appearing in the terms $t_{1}, \ldots, t_{n}$.

Proof. Suppose $\left(\bar{t}_{1}, \ldots, \bar{t}_{n}\right) \in F^{n}$ is a solution of $p \approx q$. Then we have

$$
p^{F}\left(\bar{t}_{1}, \ldots, \bar{t}_{n}\right)=q^{F}\left(\bar{t}_{1}, \ldots, \bar{t}_{n}\right),
$$

and therefore

$$
\frac{p\left(t_{1}, \ldots, t_{n}\right)}{R_{\mathbf{V}}(X)}=\frac{q\left(t_{1}, \ldots, t_{n}\right)}{R_{\mathbf{V}}(X)} .
$$

This shows that $\left(p\left(t_{1}, \ldots, t_{n}\right), q\left(t_{1}, \ldots, t_{n}\right)\right) \in R_{\mathbf{V}}(X)$ and hence by the above lemma

$$
\mathbf{V} \vDash \forall x_{1} \ldots \forall x_{m} p\left(t_{1}, \ldots, t_{n}\right) \approx q\left(t_{1}, \ldots, t_{n}\right) .
$$

The converse statement can be proved similarly.

Corollary 2. Let $\mathbf{V}$ be a variety, $X=\left\{x_{1}, \ldots, x_{n}\right\}$ and $F=F_{\mathbf{V}}(X)$. Then

$$
\operatorname{Rad}\left(\bar{x}_{1}, \ldots, \bar{x}_{n}\right)=\operatorname{Id}_{X}(\mathbf{V})
$$

where $\operatorname{Id}_{X}(\mathbf{V})$ denotes the set of identities of $\mathbf{V}$ with $X$ as the set of variables.

4.2. Finitely axiomatizable classes. We can apply the property of being equational noetherian for certain relatively free algebras, to obtain finite bases of axioms (consisting of identities) for some classes of algebras. For definition of Horn class, see [16]. In the next theorem, $\operatorname{Horn}(A)$ is used for the Horn class generated by $A$, i.e. the class of all models of Horn theory of $A$. Note that it is well-known that this class is equal to $P_{f}(A)$, where $P_{f}$ denotes the filter product. 
Theorem 3. Let $A$ be an algebra of type $\mathcal{L}$ and $\mathbf{V}=\operatorname{Var}(A)$. Let $F_{\mathbf{V}}(X)$ be equational noetherian for all finite $X$. Suppose $\mathbf{W}$ is a subclass of $\operatorname{Horn}(A)$ axiomatized by a set of identities $\Sigma \subseteq A t_{\mathcal{L}}\left(x_{1}, \ldots, x_{n}\right)$ with inside the class $\operatorname{Horn}(A)$. Then there exists a finite subset $\Sigma_{0} \subseteq \Sigma$ which axiomatizes $\mathbf{W}$ inside $\operatorname{Horn}(A)$, i.e.

$$
\mathbf{W}=\left\{B \in \operatorname{Horn}(A): B \vDash \Sigma_{0}\right\} .
$$

Proof. Suppose $\Sigma=\left\{p_{i} \approx q_{i}: i \in I\right\}$, so we have

$$
\mathbf{W}=\left\{B \in \operatorname{Horn}(A): B \vDash \bigwedge_{i \in I} \forall x_{1} \ldots \forall x_{n} p_{i} \approx q_{i}\right\}
$$

Let $X=\left\{x_{1}, \ldots, x_{n}\right\}$ and $F=F_{\mathbf{V}}(X)$. We can consider $\Sigma$ as a system of equations over $F$ and since $F$ is equational noetherian, so there exists a finite $\Sigma_{0} \subseteq \Sigma$ such that $V_{F}(\Sigma)=V_{F}\left(\Sigma_{0}\right)$. Let $I_{0}$ be the corresponding set of indices, i.e.

$$
\Sigma_{0}=\left\{p_{i} \approx q_{i}: i \in I_{0}\right\}
$$

Now, using the corollary 1 in 4.1 , for any $t_{1}, \ldots, t_{n}$, we have

$$
\mathbf{V} \vDash \bigwedge_{i \in I_{0}} \forall x_{1} \ldots \forall x_{n} p_{i}\left(t_{1}, \ldots, t_{n}\right) \approx q_{i}\left(t_{1}, \ldots, t_{n}\right),
$$

if and only if

$$
\mathbf{V} \vDash \bigwedge_{i \in I} \forall x_{1} \ldots \forall x_{n} p_{i}\left(t_{1}, \ldots, t_{n}\right) \approx q_{i}\left(t_{1}, \ldots, t_{n}\right) .
$$

Since $\mathbf{V}=\operatorname{Var}(A)$, so as a special case we have

$$
A \vDash\left(\bigwedge_{i \in I_{0}} \forall x_{1} \ldots \forall x_{n} p_{i} \approx q_{i} \Rightarrow \bigwedge_{i \in I} \forall x_{1} \ldots \forall x_{n} p_{i} \approx q_{i}\right) .
$$

This shows that for any $j \in I$, the Horn sentence

$$
\bigwedge_{i \in I_{0}} \forall x_{1} \ldots \forall x_{n} p_{i} \approx q_{i} \Rightarrow \forall x_{1} \ldots \forall x_{n} p_{j} \approx q_{j}
$$

belongs to the Horn theory of $A$. Therefore

$$
\operatorname{Th}_{H o r n}(A)+\left(\bigwedge_{i \in I_{0}} \forall x_{1} \ldots \forall x_{n} p_{i} \approx q_{i}\right) \vDash \forall x_{1} \ldots \forall x_{n} p_{j} \approx q_{j},
$$

and hence

$$
\mathbf{W}=\left\{B \in \operatorname{Horn}(A): B \vDash \bigwedge_{i \in I_{0}} \forall x_{1} \ldots \forall x_{n} p_{i} \approx q_{i}\right\},
$$

therefore $\Sigma_{0}$ is a set of axioms for $\mathbf{W}$ inside $\operatorname{Horn}(A)$. 
In the following example, we use the fact that every variety is generated by any of its infinitely generated relatively free elements. We also use the fact that the free group of the rank two, contains a free group of infinite rank as a subgroup.

Example 1. Let $\mathbf{V}$ be the variety of all groups. Clearly $\mathbf{V}=\operatorname{Var}\left(F_{2}\right)$, where $F_{2}$ is the free group of rank two. Let $n \geq 1003$ be an odd number and consider the following set of group identities

$$
\Sigma=\left\{\left[x^{p n}, y^{p n}\right]^{n} \approx 1: p=\text { prime }\right\} .
$$

Let $\mathbf{W}_{1}$ be the variety of groups axiomatized by $\Sigma$. Then, as Adian proves in [19], $\mathbf{W}_{1}$ is not finitely based, i.e. it is impossible to axiomatize it using a finite set of identities. Now, suppose

$$
\mathbf{W}=\left\{B \in \operatorname{Horn}\left(F_{2}\right): B \vDash \Sigma\right\}=\mathbf{W}_{1} \cap \operatorname{Horn}\left(F_{2}\right) .
$$

Since for any finite $X$, the free group $F(X)$ is equational noetherian, so by the above theorem $\mathbf{W}$ can be axiomatized by a finite subset of $\Sigma$. This means that there are prime numbers $p_{1}, \ldots, p_{m}$ such that

$$
\operatorname{Th}_{\text {Horn }}\left(F_{2}\right)+\left(\bigwedge_{i=1}^{m} \forall x \forall y\left[x^{p_{i} n}, y^{p_{i} n}\right]^{n} \approx 1\right) \vdash \Sigma .
$$

Hence, although $\Sigma$ is independent over $\operatorname{Id}\left(F_{2}\right)$, it is not so over the Horn theory of $F_{2}$.

\section{Some Equational noetherian Groups}

This section is marginal and it contains some results on equational noetherian groups. For the sake of generality, we consider the equations with coefficients from a fixed group $A$.

We assume that $A$ is an arbitrary group. An $A$-group is a pair $(G, \lambda)$, where $G$ is a group and $\lambda: A \rightarrow G$ is an embedding. If there is no risk of confusion, we will say that $G$ is an $A$-group, and so it contains a distinguished copy of $A$. Let $\mathcal{L}=\left(\cdot,{ }^{-1}, 1\right)$ be the language of groups and for any $a \in A$ attach a constant symbol $a$ to $\mathcal{L}$. As usual, we denote the extended language by $\mathcal{L}(A)$ and so every $A$-group $G$ becomes an algebra of type $\mathcal{L}(A)$, if we interpret $a$ as $\lambda(a)$. Note that any congruence of $G$ is in fact a normal subgroup $K$ with the property $A \cap K=1$. Through this section, we will call such a normal subgroup an $A$-ideal. We say that $G$ is noetherian if it has maximal condition on the set of $A$-ideals, i.e. any ascending chain of $A$-ideals terminates.

For a set $X=\left\{x_{1}, \ldots, x_{n}\right\}$ the free $A$-group generated by $X$ is the free product $A[X]=A * F[X]$, where $F[X]$ is the ordinary free group on $X$. We will assume that the embedding $A \hookrightarrow A[X]$ is the inclusion 
map. Any subset $S \subseteq A[X]$ corresponds to a system of equations, and if $w=w\left(x_{1}, \ldots, x_{n}, a_{1}, \ldots, a_{m}\right) \in A[X]$ then the expression $w \approx 1$ is an equation with coefficient $a_{1}, \ldots, a_{m} \in A$. Let $(G, \lambda)$ be an $A$-group. We say that $\bar{g}=\left(g_{1}, \ldots, g_{n}\right) \in G^{n}$ is a solution for this equation if

$$
w\left(g_{1}, \ldots, g_{n}, \lambda\left(a_{1}\right), \ldots, \lambda\left(a_{m}\right)\right)=1
$$

For convenience, we will write the above equality as

$$
w\left(g_{1}, \ldots, g_{n}, a_{1}, \ldots, a_{m}\right)=1
$$

As usual, we denote by $V_{G}(S)$ the algebraic set corresponding to $S$.

Theorem 4. Assume that $\mathbf{V}$ is variety of A-groups. Then all elements of $\mathbf{V}$ are equational noetherian if and only if for all finite $X$, the relatively free A-group $F_{\mathbf{V}}(X)$ is noetherian.

Proof. An arbitrary element $w R_{\mathbf{V}}(X)$ in $F_{\mathbf{V}}(X)$ will be denoted by $\bar{w}$. Let $H$ be an element of $\mathbf{V}$ and $h_{1}, \ldots, h_{n} \in H$. We define a homomorphism $\varphi: F_{\mathbf{V}}(X) \rightarrow H$ by

$$
\varphi(\bar{w})=w\left(h_{1}, \ldots, h_{n}\right)
$$

Note that this is actually a well-defined map, indeed if $\bar{w}_{1}=\bar{w}_{2}$, then $w_{1}^{-1} w_{2} \in R_{\mathbf{V}}(X)$ and so $w_{1}^{-1} w_{2} \approx 1$ is an identity in $\mathbf{V}$. Hence, we have

$$
w_{1}\left(h_{1}, \ldots, h_{n}\right)=w_{2}\left(h_{1}, \ldots, h_{n}\right) .
$$

We say that $\left(h_{1}, \ldots, h_{n}\right)$ is a solution of $\bar{w} \approx 1$ if $\varphi(\bar{w})=1$. For a subset $S \subseteq F_{\mathbf{V}}(X)$, we define

$$
V_{H}(S)=\left\{\left(h_{1}, \ldots, h_{n}\right) \in H^{n}: \forall \bar{w} \in S \quad w\left(h_{1}, \ldots, h_{n}\right)=1\right\} .
$$

Note that although $S$ is not an ordinary system of equations here, $V_{H}(S)$ is an ordinary algebraic set in $H^{n}$.

Now we prove the theorem. The noetherianity of $F_{\mathbf{V}}(X)$ means the max property for $A$-ideals. So, first let for any finite $X$, the group $F_{\mathbf{V}}(X)$ has max on $A$-ideals. Let $H \in \mathbf{V}$ and $S \subseteq A[X]$. Let $R$ be the normal closure of $S$ in $A[X]$. Clearly $V_{H}(S)=V_{H}(R)$, indeed every element of $R$ has the form $\prod_{i=1}^{N} u_{i} w_{i}^{ \pm 1} u_{i}^{-1}$, where $w_{i} \in S$ and $u_{i} \in A[X]$. We will prove that there is a finite subset $R_{0} \subseteq R$ such that $\left.V_{H}(R)=V_{(} R_{0}\right)$. Assume that we done. Let

$$
R_{0}=\left\{v_{1}, \ldots, v_{k}\right\}
$$

Then for any $i$, we have

$$
v_{i}=\prod_{j=1}^{N_{i}} u_{i j} w_{i j}^{ \pm 1} u_{i j}^{-1},
$$


with $u_{i j} \in A[X]$ and $w_{i j} \in S$. Let

$$
S_{0}=\left\{w_{i j}: 1 \leq i \leq k, 1 \leq j \leq N_{i}\right\} .
$$

Then $S_{0} \subseteq S$ and $V_{H}(S)=V_{H}\left(S_{0}\right)$. Therefore, we are going to prove the existence of $R_{0}$. Let

$$
\bar{S}=\left\{\bar{w} \in F_{\mathbf{V}}(X): w \in S\right\} .
$$

Assume that $\bar{R}$ is the normal closure of $\bar{S}$ in $F_{\mathbf{V}}(X)$. Note that we have

$$
\bar{R}=\{\bar{w}: w \in R\} .
$$

On the other hand we have

$$
V_{H}(S)=V_{H}(R)=V_{H}(\bar{R})=V_{H}(\bar{S}) .
$$

Now, there are two cases:

i) $A \cap \bar{R} \neq 1$. Then there exists an element $1 \neq a \in A \cap \bar{R}$. Note that $\bar{a} \neq 1$ and so $V_{H}(\bar{R})=\emptyset$. Hence we can put $R_{0}=\{a\} \subseteq R$ in this case.

ii) $A \cap \bar{R}=1$. In this case $\bar{R}$ is an $A$-ideal of $F_{\mathbf{V}}(X)$ and so it is finitely generated as an $A$-ideal. Hence there exists a finite $\bar{R}_{0} \subseteq \bar{R}$, generating $\bar{R}$. We have $V_{H}(\bar{R})=V_{H}\left(\bar{R}_{0}\right)$. Suppose $R_{0}$ is a set of pre-images of elements of $\bar{R}_{0}$. Then $V_{H}\left(\bar{R}_{0}\right)=V_{H}\left(R_{0}\right)$ and so $V_{H}(R)=V_{H}\left(R_{0}\right)$. This proves that $H$ is equational noetherian.

Now assume that every element of $\mathbf{V}$ is equational noetherian. Let $X=\left\{x_{1}, \ldots, x_{n}\right\}$ be a finite set. We prove that $F_{\mathbf{V}}(X)$ is noetherian. Assume that $K$ is an arbitrary $A$-ideal in $F_{\mathbf{V}}(X)$ and $H=F_{\mathbf{V}}(X) / K$. Then $H$ is an $A$-group belonging to $\mathbf{V}$. Let $\bar{w} \in K$. Then we have

$$
\begin{aligned}
w\left(\bar{x}_{1} K, \ldots, \bar{x}_{n} K\right) & =w\left(\bar{x}_{1}, \ldots, \bar{x}_{n}\right) K \\
& =\bar{w} K \\
& =K .
\end{aligned}
$$

This shows that the point $\left(\bar{x}_{1} K, \ldots, \bar{x}_{n} K\right) \in H^{n}$ belongs to $V_{H}(\bar{w} \approx 1)$. Conversely, if $\bar{w} \in F_{\mathbf{V}}(X)$ and $\left(\bar{x}_{1} K, \ldots, \bar{x}_{n} K\right)$ is a solution of $\bar{w} \approx 1$, then $\bar{w} \in K$. We conclude that

$$
\bar{w} \in K \Leftrightarrow\left(\bar{x}_{1} K, \ldots, \bar{x}_{n} K\right) \in V_{H}(\bar{w} \approx 1) .
$$

Now, assume that $K_{1} \varsubsetneqq K_{2} \varsubsetneqq K_{3} \varsubsetneqq \cdots$ is a proper chain of $A$-ideals in $F_{\mathbf{V}}(X)$. For any $i$, let $\bar{w}_{i} \in K_{i+1} \backslash K_{i}$ and let $L_{i}$ be the normal closure of the set $K_{i} \cup\left\{\bar{w}_{i}\right\}$. We have $K_{i} \varsubsetneqq L_{i} \subseteq K_{i+1}$. Let $H_{i}=F_{\mathbf{V}}(X) / K_{i}$ 
and $H=\prod_{i=1}^{\infty} H_{i}$. By assumption $H$ is equational noetherian. For any $i$, we have $H_{i} \leq H$ and so

$$
\left(\bar{x}_{1} K_{i}, \ldots, \bar{x}_{n} K_{i}\right) \in V_{H_{i}}\left(K_{i}\right) \subseteq V_{H}\left(K_{i}\right) .
$$

On the other hand $\left(\bar{x}_{1} K_{i}, \ldots, \bar{x}_{n} K_{i}\right)$ does not belong to $V_{H}\left(L_{i}\right)$, since otherwise $\left(\bar{x}_{1} K_{i}, \ldots, \bar{x}_{n} K_{i}\right) \in V_{H_{i}}\left(\bar{w}_{i} \approx 1\right)$ which implies by $(*)$ that $\bar{w}_{i} \in K_{i}$. Hence we have

$$
V_{H}\left(K_{1}\right) \supsetneq V_{H}\left(L_{1}\right) \supseteq V_{H}\left(K_{2}\right) \supsetneq V_{H}\left(L_{2}\right) \supseteq \cdots
$$

and hence we have the following proper chain of algebraic sets in $H^{n}$,

$$
V_{H}\left(K_{1}\right) \supsetneq V_{H}\left(K_{2}\right) \supsetneq V_{H}\left(K_{3}\right) \supsetneq \cdots
$$

which is a contradiction.

Recall that an arbitrary group $A$ is equational noetherian if and only if it is equational noetherian as an $A$-group. A well-known theorem of $\mathrm{P}$. Hall says that any finitely generated metabelian group $A$ has $\max -n$ property, so we can use the above theorem to prove the next result.

Corollary 3. Every finitely generated metabelian group is equational noetherian.

Proof. Let $A$ be a finitely generated metabelian group and $\mathbf{V}=\operatorname{Var}_{A}(A)$ be the variety of $A$-groups generated by $A$. Since $A$ satisfies the identity $\left[\left[x_{1}, x_{2}\right],\left[x_{3}, x_{4}\right]\right] \approx 1$ and this is also an identity in the language $\mathcal{L}(A)$, so every element of $\mathbf{V}$ is metabelian. Now, for a finite set $X$, the group $F_{\mathbf{V}}(X)$ is metabelian and finitely generated since it is generated by $A \cup X$ as an ordinary group. So by a well-known theorem of P. Hall, it has max $-n$ property. Therefore, it has also max on $A$-ideals. So, by the above theorem, every element of $\mathbf{V}$, and specially $A$ is equational noetherian.

The above result is not new, indeed it is known already by the authors of [1]. Using Hilbert's basis theorem, it is proved that every linear group over a noetherian ring is equational noetherian (see [1]). On the other hand, a theorem of Remeslennikov, says that a finitely generated metabelian group has a faithful representation over a ring which is a direct product of finitely many fields. This shows that every finitely generated metabelian group is equational noetherian. Our method is different in some features: we don't need the result of Remeslennikov, we use Theorem 4 and the above mentioned theorem of Hall. However, our proof applies again Hilbert's basis theorem, because the proof of Hall's theorem depends on it. 
We obtain a second corollary of the theorem 4, which relates equational noetherian $A$-group and finitely based varieties. Note that a variety is finitely based, if it can be defined by a finite set of identities. A variety has finite axiomatic rank, if it can be defined by a finite number of variables.

Corollary 4. Let $\mathbf{V}$ be a variety of A-groups which has finite axiomatic rank. If all elements of $\mathbf{V}$ are equational noetherian, then $\mathbf{V}$ is finitely based.

Proof. Let $X=\left\{x_{1}, \ldots, x_{n}\right\}$ be the set of variables which we need to define $\mathbf{V}$ and $R=\operatorname{Id}_{X}(\mathbf{V})$. It is easy to see that

$$
R=\operatorname{Rad}\left(\bar{x}_{1}, \ldots, \bar{x}_{n}\right),
$$

and so it is finitely generated as an $A$-ideal of $F_{\mathbf{V}}(X)$ (as we assumed that all elements of $\mathbf{V}$ are equational noetherian). This proves that $\mathbf{V}$ is finitely based.

With some minor changes, Theorem 4 will be given also for general types of algebras in the section 6 (see subsections 6.2 and 6.3).

In the next theorem, we will concentrate on $A$ as an $A$-group. We give a sufficient condition under which a group $A$ is equational noetherian. An $A$-group $G$ is called finitely cogenerated, if for any family $\left\{K_{i}\right\}_{i \in I}$ of $A$-ideals, the assumption $\bigcap_{i \in I} K_{i}=1$ implies that there is a finite subset $I_{0} \subseteq I$ such that $\bigcap_{i \in I_{0}} K_{i}=1$.

Theorem 5. Let $A$ be a group and $\mathbf{V}=\operatorname{Var}_{A}(A)$ be the variety generated by $A$ as an algebra of type $\mathcal{L}(A)$. Assume that for all $m \geq 1$, all finitely generated subgroup of $A^{m}$ have $\max -n$. Assume also for all finite $X$, the group $F_{\mathbf{V}}(X)$ is finitely cogenerated. Then $A$ is equational noetherian.

Proof. Recall that $\mathbf{V}=\operatorname{Var}_{A}(A)$ is the variety of $A$-groups generated by $A$. For any set $X$, we have

$$
R_{\mathbf{V}}(X)=\bigcap\left\{R \unlhd A[X]: A \cap R=1, \frac{A[X]}{R} \hookrightarrow A\right\},
$$

and

$$
F_{\mathbf{V}}(X)=\frac{A[X]}{R_{\mathbf{V}}(X)}
$$

Now assume that

$$
\mathcal{C}=\left\{R \unlhd A[X]: A \cap R=1, \frac{A[X]}{R} \hookrightarrow A\right\} .
$$


Let $R \in \mathcal{C}$. Then we have $R / R_{\mathbf{V}}(X) \unlhd F_{\mathbf{V}}(X)$ and this is an $A$-ideal since $A \cap R=1$. Now we have

$$
\bigcap_{R \in \mathcal{C}} \frac{R}{R_{\mathbf{V}}(X)}=1
$$

so by the assumption of finitely cogeneratedness of $F_{\mathbf{V}}(X)$, there are finitely many elements $R_{1}, \ldots, R_{m}$ in $\mathcal{C}$ such that

$$
R_{\mathbf{V}}(X)=\bigcap_{i=1}^{m} R_{i}
$$

Hence we have

$$
F_{\mathbf{V}}(X)=\frac{A[X]}{\bigcap_{i=1}^{m} R_{i}} \hookrightarrow \prod_{i=1}^{m} \frac{A[X]}{R_{i}} \hookrightarrow A^{m} .
$$

By the assumption, $F_{\mathbf{V}}(X)$ has $\max -n$, so it has also max on $A$-ideals. Hence every element of $\mathbf{V}$, specially $A$ itself, is equational noetherian.

If we apply this theorem for the case of a locally finite group $A$, then the first assumption on the finitely generated subgroups of $A^{m}$ will be automatically fulfilled. So we obtain our last result.

Corollary 5. Let $A$ be a locally finite group and $\mathbf{V}=\operatorname{Var}_{A}(A)$ be the variety generated by $A$ as an algebra of type $\mathcal{L}(A)$. Assume also for all finite $X$, the group $F_{\mathbf{V}}(X)$ is finitely cogenerated. Then $A$ is equational noetherian.

Proof. Let $\mathbf{V}=\operatorname{Var}_{A}(A)$. Since $A$ is locally finite, so for all $m \geq$ 1 , every subgroup of $A^{m}$ is finite so it has $\max -n$. Now, by the assumption, for all finite $X$, the group $F_{\mathbf{V}}(X)$ is finitely cogenerated. So we conclude that $A$ is equational noetherian.

\section{Equational Artinian algebras}

We say that an algebra $A$ is equational Artinian if every ascending chain of algebraic sets over $A$ terminates. In this section, we investigate some properties of equational Artinian algebras.

6.1. A-radicals. One can ask about the existence of an equational condition, equivalent to being equational Artinian. In this subsection, we will show that the correct condition is not in terms of equations, but rather it can be formulated in terms of radical ideals. We will prove 
that $A$ is equational Artinian, iff for any $n$ and $E \subseteq A^{n}$, there exists a finite subset $E_{0} \subseteq E$ such that

$$
\operatorname{Rad}(E)=\operatorname{Rad}\left(E_{0}\right) .
$$

Note that this condition is in some sense the dual condition of being equational noetherian. First we recall a definition.

Definition 5. Let $A$ be an algebra and $E \subseteq A^{n}$, for some $n$. Then $\operatorname{Rad}(E)$ is called an $A$-radical ideal of the term algebra $T_{\mathcal{L}}\left(x_{1}, \ldots, x_{n}\right)$.

Theorem 6. For an algebra $A$, the following conditions are equivalent;

$i$ - For any $n$ and $E \subseteq A^{n}$, there exists a finite subset $E_{0} \subseteq E$ such that

$$
\operatorname{Rad}(E)=\operatorname{Rad}\left(E_{0}\right)
$$

ii- Every descending chain of A-radical ideals terminates.

iii- $A$ is equational Artinian.

Proof. We first show that $i \Leftrightarrow i i$. Suppose $A$ satisfies $i$. Let

$$
\operatorname{Rad}\left(E_{1}\right) \supseteq \operatorname{Rad}\left(E_{2}\right) \supseteq \operatorname{Rad}\left(E_{3}\right) \supseteq \cdots
$$

be a descending chain of $A$-radicals, with $E_{i} \subseteq A^{n}$. Let

$$
E=\bigcup_{i=1}^{\infty} V_{A}\left(\operatorname{Rad}\left(E_{i}\right)\right)
$$

By $i$, there exists a finite $E_{0} \subseteq E$ such that $\operatorname{Rad}(E)=\operatorname{Rad}\left(E_{0}\right)$. Since $E_{0}$ is finite, so there is $k \geq 1$ with

$$
E_{0} \subseteq V_{A}\left(\operatorname{Rad}\left(E_{k}\right)\right)
$$

Hence, we have

$$
\operatorname{Rad}(E)=\operatorname{Rad}\left(E_{0}\right) \supseteq \operatorname{Rad}\left(E_{k}\right) .
$$

On the other hand

$$
\operatorname{Rad}(E)=\bigcap_{i=1}^{\infty} \operatorname{Rad}\left(E_{i}\right) \subseteq \operatorname{Rad}\left(E_{k}\right),
$$

so the chain terminates. Now, suppose $A$ has the property ii. We prove $i$. Let $E \subseteq A^{n}$. Choose an arbitrary $c_{1} \in E$. If we have $\operatorname{Rad}(E)=\operatorname{Rad}\left(\left\{c_{1}\right\}\right)$, then we done. So, let $\operatorname{Rad}(E) \varsubsetneqq \operatorname{Rad}\left(\left\{c_{1}\right\}\right)$. This shows that there is an equation $p \approx q$ such that

$$
(p, q) \in \operatorname{Rad}\left(\left\{c_{1}\right\}\right) \backslash \operatorname{Rad}(E) .
$$

Hence there is a $c_{2} \in E$ with $p^{A}\left(c_{2}\right) \neq q^{A}\left(c_{2}\right)$. Now, if $\operatorname{Rad}(E)=$ $\operatorname{Rad}\left(\left\{c_{1}, c_{2}\right\}\right)$, then the result follows, otherwise we can continue this 
argument to obtain a non-terminating descending chain of $A$-radicals. Therefore we proved $i \Leftrightarrow i i$. We prove $i i \Rightarrow i i i$. Suppose

$$
Y_{1} \subseteq Y_{2} \subseteq Y_{3} \subseteq \cdots
$$

is a chain of algebraic sets in $A^{n}$. We have $Y_{i}=V_{A}\left(S_{i}\right)$, for some system $S_{i}$. Now,

$$
\operatorname{Rad}\left(Y_{1}\right) \supseteq \operatorname{Rad}\left(Y_{2}\right) \supseteq \operatorname{Rad}\left(Y_{3}\right) \supseteq \cdots
$$

is a descending chain of $A$-radicals and so it terminates, i.e. there is $k$ such that

$$
\operatorname{Rad}\left(Y_{k}\right)=\operatorname{Rad}\left(Y_{k+1}\right)=\cdots
$$

This shows that

$$
V_{A}\left(\operatorname{Rad}\left(Y_{k}\right)\right)=V_{A}\left(\operatorname{Rad}\left(Y_{k+1}\right)\right)=\cdots
$$

and hence

$$
Y_{k}=Y_{k+1}=\cdots .
$$

Therefore $A$ is equational Artinian. Finally, we prove $i i i \Rightarrow i i$. Let

$$
\operatorname{Rad}\left(E_{1}\right) \supseteq \operatorname{Rad}\left(E_{2}\right) \supseteq \operatorname{Rad}\left(E_{3}\right) \supseteq \cdots
$$

be a descending chain of $A$-radicals with $E_{i} \subseteq A^{n}$. Then we have the following chain of algebraic sets;

$$
E_{1}^{a c} \subseteq E_{2}^{a c} \subseteq E_{3}^{a c} \subseteq \cdots,
$$

and by iii this chain terminates. So, the chain of $A$-radicals already terminates.

As we saw in the section 3 , an algebra $A$ is equational noetherian if and only if every subset of $A^{n}$ is compact. We have a similar statement for the case of equational Artinian domains. A subset of a topological space is contra-compact, if every covering of it by closed sets has a finite subcover. In literature, this type of subset are usually called strongly $S$-closed. However, we prefer to use the term "contra-compact".

Theorem 7. An equational domain $A$ is equational Artinian, iff for any $n$, every subset of $A^{n}$ is contra-compact.

Proof. Let a domain $A$ be equational Artinian. Let $C \subseteq A^{n}$ and

$$
C \subseteq \bigcup_{i \in I} C_{i}
$$

be a covering of $C$ by closed subsets. Suppose $i_{0} \in I$ be arbitrary. If $C \subseteq C_{i_{0}}$, then we have a finite subcover. Otherwise there is $i_{1} \in I$ such that $C_{i_{1}}$ is not contained in $C_{i_{0}}$ and $C \cap C_{i_{1}} \neq \emptyset$. If we have 
$C \subseteq C_{i_{0}} \cup C_{i_{1}}$, then we have a finite subcover, otherwise, repeating this process, we obtain a chain

$$
C_{i_{0}} \varsubsetneqq C_{i_{0}} \cup C_{i_{1}} \varsubsetneqq C_{i_{0}} \cup C_{i_{1}} \cup C_{i_{2}} \varsubsetneqq \cdots .
$$

Since $A$ is a domain, all terms of this chain are algebraic sets, and this violates the assumption of being equational Artinian for $A$. Hence every subset of $A^{n}$ is contra-compact.

Conversely, let every subset of $A^{n}$ be contra-compact and $E \subseteq A^{n}$. Since $E$ is contra-compact, there is a finite $E_{0} \subseteq E$, which is dense in $E$, i.e. $\bar{E}_{0}=E$. Since $A$ is domain, so we have $E_{0}^{a c}=E$. Hence we have

$$
\operatorname{Rad}\left(E_{0}\right)=\operatorname{Rad}\left(E_{0}^{a c}\right)=\operatorname{Rad}(E) .
$$

By the previous theorem $A$ is equational Artinian.

There is also another topological characterization of equational Artinian algebras under some additional conditions, which we discuss it in the final section (see the section 6). As a simple application, we discuss a basic property of equational Artinian groups. Let $G$ be a group and $B \subseteq G^{n}$. We say that $B$ is an identity base of $G$, if for all $w \in F\left(x_{1}, \ldots, x_{n}\right), w_{B}=1$ implies $(w \approx 1) \in \operatorname{Id}_{G}\left(x_{1}, \ldots, x_{n}\right)$. In other words, if all elements of $B$ satisfy the role $w \approx 1$, then $w \approx 1$ is an identity in $G$.

Corollary 6. Every equational Artinian group has a finite identity base.

Proof. We have

$$
\operatorname{Id}_{G}\left(x_{1}, \ldots, x_{n}\right)=\operatorname{Rad}\left(G^{n}\right) .
$$

Since $G$ is equational Artinian, so there is a finite $B \subseteq G^{n}$ such that $\operatorname{Rad}\left(G^{n}\right)=\operatorname{Rad}(B)$, and so $B$ is an identity base of $G$.

6.2. Relative systems of equations. Let $\mathbf{V}$ be a variety of algebras. In the next subsection, we will show that the relative free algebra $F_{\mathbf{V}}(X)$ has descending chain condition on its ideals, if and only if every element of $V$ is equational Artinian. We will do this in a more general context.

In the sequel, we assume that $A$ is an algebra containing a trivial subalgebra. Suppose $\mathbf{V}$ is a pre-variety of $A$-algebras. As before, let $X$ be a finite set of variables. Suppose $R_{\mathbf{V}}(X)$ is the smallest $A$-congruence with the property $T_{\mathcal{L}(A)}(X) / R_{\mathbf{V}}(X) \in \mathbf{V}$. Let

$$
F_{\mathbf{V}}(X)=\frac{T_{\mathcal{L}(A)}(X)}{R_{\mathbf{V}}(X)} .
$$


As before, we denote an arbitrary element of $F_{\mathbf{V}}(X)$ by $\bar{t}$, where $t$ is a term in $\mathcal{L}(A)$.

Suppose now, $B \in \mathbf{V}$ and $\left(b_{1}, \ldots, b_{n}\right) \in B^{n}$. We know that there exists a homomorphism $\varphi: F_{\mathbf{V}}(X) \rightarrow B$ such that

$$
\varphi(\bar{p})=p^{B}\left(b_{1}, \ldots, b_{n}\right) .
$$

Therefore, if $\bar{p}_{1}=\bar{p}_{2}$, then $p_{1}^{B}\left(b_{1}, \ldots, b_{n}\right)=p_{2}^{B}\left(b_{1}, \ldots, b_{n}\right)$. This shows that the following definition has no ambiguity.

Definition 6. A V-equation is an expression of the form $\bar{p} \approx \bar{q}$, where $p$ and $q$ are terms in the language $\mathcal{L}(A)$. If $B$ is an A-algebra and $\left(b_{1}, \ldots, b_{n}\right)$ is an element of $B^{n}$, we say that $\left(b_{1}, \ldots, b_{n}\right)$ is a solution of $\bar{p} \approx \bar{q}$, if $p^{B}\left(b_{1}, \ldots, b_{n}\right)=q^{B}\left(b_{1}, \ldots, b_{n}\right)$.

Let $S$ be a system of $\mathbf{V}$-equations. The set of all solutions of elements of $S$, will be denoted by $V_{B}^{\mathbf{V}}(S)$. The following observation shows that this is an ordinary algebraic set. Let $S^{\prime}$ be the set of all equations $p \approx q$ such that $\bar{p} \approx \bar{q}$ belongs to $S$. Then it can be easily verified that

$$
V_{B}^{\mathbf{V}}(S)=V_{B}\left(S^{\prime}\right) \text {. }
$$

Therefore, in the sequel we will denote the algebraic set $V_{B}^{\mathbf{V}}(S)$ by the same notation $V_{B}(S)$. The Zariski topology arising from algebraic sets relative to the pre-variety $\mathbf{V}$ is the same as the ordinary Zariski topology. If $Y \subseteq B^{n}$, we define

$$
\operatorname{Rad}_{B}^{\mathbf{V}}(Y)=\left\{\bar{p} \approx \bar{q}: \forall \bar{b} \in Y p^{B}\left(b_{1}, \ldots, b_{n}\right)=q^{B}\left(b_{1}, \ldots, b_{n}\right)\right\} .
$$

The quotient algebra

$$
\Gamma_{\mathbf{V}}(Y)=\frac{F_{\mathbf{V}}(X)}{\operatorname{Rad}_{B}^{\mathbf{V}}(Y)}
$$

is the $\mathbf{V}$-coordinate algebra of $Y$. Again, it is easy to see that $\Gamma_{\mathbf{V}}(Y) \cong$ $\Gamma(Y)$.

6.3. Chain conditions on the ideals of relatively free algebras. An algebra $B$ will be called noetherian (Artinian), if any ascending (descending) chain of ideals in $B$ terminates. In the case of $A$-algebras, we restrict ourself to $A$-ideals. A congruence $R$ in $B$ is called $A$-ideal, if for all $a_{1}, a_{2} \in A$, the assumption $\left(a_{1}, a_{2}\right) \in R$ implies $a_{1}=a_{2}$. The following theorem is is very similar to Theorem 4 of the section 5 , and so we don't prove it here (for a proof one can see also [20]).

Theorem 8. Let $\mathfrak{Y}$ be a variety of algebras of type $\mathcal{L}$ and $A \in \mathfrak{Y}$ containing a trivial subalgebra. Let $\mathbf{V}=\mathfrak{Y}_{A}$ be the class of elements of $\mathfrak{Y}$ which are A-algebra. Then for any finite $X$, the relatively free algebra 
$F_{\mathbf{V}}(X)$ is noetherian if and only if every $B \in \mathbf{V}$ is A-equationally noetherian.

Note that an $A$-algebra $B$ is called $A$-equational noetherian ( $A$ equational Artinian), if it is equational noetherian (equational Artinian) as an algebra of type $\mathcal{L}(A)$.

We are now ready to prove the analogue of the above theorem for the property of being Artinian.

Theorem 9. Let $\mathfrak{Y}$ be a variety of algebras of type $\mathcal{L}$ and $A \in \mathfrak{Y}$ containing a trivial subalgebra. Let $\mathbf{V}=\mathfrak{Y}_{A}$ be the class of elements of $\mathfrak{Y}$ which are A-algebra. Then for any finite $X$, the relatively free algebra $F_{\mathbf{V}}(X)$ is Artinian, if and only if every $B \in \mathbf{V}$ is A-equationally Artinian.

Proof. The main idea of the proof is the same as in Theorem 4. First, suppose that $F_{\mathbf{V}}(X)$ is Artinian and $B \in \mathbf{V}$. Let

$$
Y_{1} \subseteq Y_{2} \subseteq Y_{3} \subseteq \cdots
$$

be a chain of algebraic sets in $B^{n}$. Then we have

$$
\operatorname{Rad}\left(Y_{1}\right) \supseteq \operatorname{Rad}\left(Y_{2}\right) \supseteq \operatorname{Rad}\left(Y_{3}\right) \supseteq \cdots,
$$

which is a chain of $A$-ideals in $F_{\mathbf{V}}(X)$. So, it terminates and hence, there is $m$ such that

$$
\operatorname{Rad}\left(Y_{m}\right)=\operatorname{Rad}\left(Y_{m+1}\right)=\cdots .
$$

This implies that $Y_{m}=Y_{m=1}=\cdots$ and therefore $B$ is equational Artinian. Now, suppose $B$ is equational Artinian for all $B \in \mathbf{V}$. If $R$ is an $A$-ideal in $F_{\mathbf{V}}(X)$, we put $B(R)=F_{\mathbf{V}}(X) / R$, which is belong to $\mathbf{V}$. It is easy to see that $(\bar{p}, \bar{q}) \in R$ if and only if

$$
\left(\bar{x}_{1} / R, \ldots, \bar{x}_{n} / R\right) \in V_{B(R)}(\bar{p} \approx \bar{q}) .
$$

Now, let

$$
R_{1} \supsetneq R_{2} \supsetneq R_{3} \supsetneq \cdots
$$

be a proper descending chain of $A$-ideals in $F_{\mathbf{V}}(X)$. Note that in the same time, $R_{i}$ is a system of $\mathbf{V}$-equations. Suppose $\left(\bar{p}_{i}, \bar{q}_{i}\right) \in R_{i} \backslash R_{i+1}$. Suppose also that $T_{i}$ is the $A$-ideal generated by the set $R_{i+1}+\left(\bar{p}_{i}, \bar{q}_{i}\right)$. Then we have

$$
R_{i+1} \varsubsetneqq T_{i} \varsubsetneqq R_{i}
$$

Suppose $B_{i}=B\left(R_{i}\right)$ and $B=\prod_{i} B_{i}$. So $B \in \mathbf{V}$. Hence it is equational Artinian. Note that, since $A$ contains trivial algebra, so $B_{i} \leq B$. Hence

$$
U=\left(\bar{x}_{1} / R_{i+1}, \ldots, \bar{x}_{n} / R_{i+1}\right) \in V_{B_{i+1}}\left(R_{i+1}\right) \subseteq V_{B}\left(R_{i+1}\right) .
$$


But, $U$ does not belong to $V_{B}\left(T_{i}\right)$, since otherwise, $U \in V_{B}\left(\bar{p}_{i} \approx \bar{q}_{i}\right)$ which implies that $\left(\bar{p}_{i}, \bar{q}_{i}\right) \in R_{i+1}$. Therefore, we have

$$
V_{B}\left(R_{i}\right) \subseteq V_{B}\left(T_{i}\right) \varsubsetneqq V_{B}\left(R_{i+1}\right),
$$

so the chain $V_{B}\left(R_{1}\right) \varsubsetneqq V_{B}\left(R_{2}\right) \varsubsetneqq V_{B}\left(R_{3}\right) \varsubsetneqq \cdots$ is a proper chain of algebraic sets, which is a contradiction.

6.4. Hilbert's basis theorem. A universal algebraic version of the Hilbert's basis theorem is given in [20]. In this subsection, we discuss briefly some results on this item. Suppose $\mathcal{L}$ is an algebraic language and $\mathfrak{Y}$ is a variety of algebras of type $\mathcal{L}$. Let $A \in \mathfrak{Y}$ and $\mathbf{V}=\mathfrak{Y}_{A}$ be the class of all elements of $\mathfrak{Y}$ which are $A$-algebra. If $A$ has maximal property on its ideals, is the algebra $F_{\mathbf{V}}(X)$ noetherian?

Example 2. Let $\mathcal{L}=(0,1,+, \times)$ be the language of unital rings and $\mathfrak{Y}$ be the variety of all commutative rings with unite element. Let $A \in \mathfrak{Y}$ and $\mathbf{V}=\mathfrak{Y}_{A}$. If $X=\left\{x_{1}, \ldots, x_{n}\right\}$, then $F_{\mathbf{V}}(X)=A\left[x_{1}, \ldots, x_{n}\right]$ and hence Hilbert's basis theorem is valid in this case.

Example 3. Let $\mathcal{L}=\left(e,^{-1}, \cdot\right)$ be the language of groups. Let $\mathfrak{Y}$ be the variety of groups. Let $A$ be any group and $\mathbf{V}=\mathfrak{Y}_{A}$. Then $F_{\mathbf{V}}(X)=A *$ $F(X)$. We show that $F_{\mathbf{V}}(X)$ is not noetherian even if $A$ has maximal property on its normal subgroups (max-n). Consider the BaumslagSolitar group

$$
B_{m, n}=\left\langle a, t: t a^{m} t^{-1}=a^{n}\right\rangle,
$$

where $m, n \geq 1$ and $m \neq n$. Then, as is proved in [1], this group is not equationally noetherian. Let $B=A * B_{m, n}$. Then $B$ is an $A$ group which is not $A$-equationally noetherian. So, by the theorem 8 of the previous subsection, $A * F(X)$ is not noetherian, Hilbert's basis theorem fails.

Example 4. Let $\mathfrak{Y}$ be the variety of abelian groups and $A \in \mathfrak{Y}$ be finitely generated. Suppose $\mathbf{V}=\mathfrak{Y}_{A}$. Then it is easy to see that $F_{\mathbf{V}}(X)=A \times F_{a b}(X)$, where $F_{a b}(X)$ is the free abelian group generated by $X$. So, $F_{\mathbf{V}}(X)=A \times \mathbb{Z}^{n}$. As a $\mathbb{Z}$-module, clearly $A \times \mathbb{Z}^{n}$ is noetherian, so Hilbert's basis theorem is true for any finitely generated abelian group $A$ in the variety of abelian groups. As a result, every abelian group $B$ containing $A$ is A-equationally noetherian.

As we mentioned above, if $A \leq B$ and $B$ is not equationally noetherian, then it is also not $A$-equationally noetherian. So, let $\mathfrak{Y}$ be a variety of algebras and $A \in \mathfrak{Y}$. Let $\mathbf{V}=\mathfrak{Y}_{A}$. If there exists an element $B \in \mathfrak{Y}$ which is not equationally noetherian, then by our theorem, $F_{\mathbf{V}}(X)$ is 
not noetherian, so we never have a version of Hilbert's basis theorem for the variety $\mathfrak{Y}$.

Example 5. Let $\mathfrak{Y}$ be the variety of nilpotent groups of class at most c. If $A \in \mathfrak{Y}$ and $\mathbf{V}=\mathfrak{Y}_{A}$ and $B \in \mathfrak{Y}$ is not finitely generated, then by [5], $B$ is not equationally noetherian and hence $F_{\mathbf{V}}(X)$ is not noetherian.

6.5. Examples of equational Artinian algebras. In this subsection, we give some examples of the equational Artinian algebras.

Example 6. Suppose $A$ is an equational Artinian algebra (for example, a finite algebra). Then for any set $I$, the algebra $A^{I}$ is also equational Artinian. This is true, because for any equation $p \approx q$, there is a natural bijection between the sets $V_{A}(p \approx q)^{I}$ and $V_{A^{I}}(p \approx q)$, given by

$$
\left(a_{i}^{1}, \ldots, a_{i}^{n}\right)_{i \in I} \mapsto\left(\left(a_{i}^{1}\right)_{i \in I}, \ldots,\left(a_{i}^{n}\right)_{i \in I}\right) .
$$

Hence, any chain

$$
V_{A^{I}}\left(S_{1}\right) \subseteq V_{A^{I}}\left(S_{2}\right) \subseteq V_{A^{I}}\left(S_{3}\right) \subseteq \cdots
$$

becomes

$$
V_{A}\left(S_{1}\right)^{I} \subseteq V_{A}\left(S_{2}\right)^{I} \subseteq V_{A}\left(S_{3}\right)^{I} \subseteq \cdots,
$$

and consequently, we obtain a chain

$$
V_{A}\left(S_{1}\right) \subseteq V_{A}\left(S_{2}\right) \subseteq V_{A}\left(S_{3}\right) \subseteq \cdots,
$$

which terminates.

Example 7. Let $R$ be a noetherian ring and $A=(R,+,-)$. Then $A$ is equational Artinian. To prove this, let $p=a_{1} x_{1}+\cdots+a_{n} x_{n}$ with $a_{i} \in \mathbb{Z}$, be a term. We show that $V_{A}(p \approx 0)$ is a submodule of $R^{n}$. Clearly, this algebraic set is closed under addition, so let $\lambda \in R$. Then the map $\alpha_{\lambda}: R \rightarrow R, \alpha_{\lambda}(x)=\lambda x$, is a homomorphism of $A$. Since every algebraic set is invariant under homomorphisms, so we have $\lambda V_{A}(p \approx$ $0) \subseteq V_{A}(p \approx 0)$. This shows that for any system $S$, the algebraic set $V_{A}(S)$ is a submodule of $R^{n}$ ( $n$ is the number of indeterminate in $S$ ). But $R^{n}$ is noetherian and hence $A$ is equational Artinian.

Example 8. Let $R$ be a noetherian ring and $\Lambda=\left(\lambda_{1}, \ldots, \lambda_{m}\right)$ be a tuple of elements of $R$ with $\sum_{i} \lambda_{i}=1$. Define an m-ary operation

$$
p_{\Lambda}\left(x_{1}, \ldots, x_{m}\right)=\sum_{i} \lambda_{i} x_{i}
$$

Then by a similar argument as in the previous example, we see that $A=\left(R, p_{\Lambda}\right)$ is equational Artinian. If we assume that $R$ is noetherian but not Artinian ring, then we obtain example of an equational Artinian algebra which is not equational noetherian. 
Example 9. It can be shown that only finite fields are equational Artinian in the language of rings $\mathcal{L}=(+, \cdot, 0,1)$.

\section{Other tyPes of EQUATIONAL CONDITIONS}

During this article, we saw many cases of equational conditions in the universal algebraic geometry. It is easy to find new kinds of equational conditions. In this final section, we give two more examples of this kind of conditions.

Let $A$ be an algebra of type $\mathcal{L}$. A system $S$ is called $A$-independent if for any finite subset $S_{0} \subseteq S$, there exists an equation $(p \approx q) \in S_{0}$, such that $V_{A}\left(S_{0}\right) \varsubsetneqq V_{A}\left(S_{0} \backslash p \approx q\right)$.

Theorem 10. For any algebra $A$ and any system $S$, there exists an $A$-independent subsystem $S^{\prime}$ equivalent to $S$ over $A$.

Proof. Suppose $A$ and $S$ are given and $\mathcal{F}$ is the collection of all $A$ independent subsets of $S$. Using Zorn's lemma, we show that $\mathcal{F}$ has a maximal element. Let $\left\{T_{\alpha}\right\}_{\alpha} \subseteq \mathcal{F}$ be a chain. Let $T=\bigcup T_{\alpha}$ and $T^{0} \subseteq T$ be finite. Then $T^{0} \subseteq T_{\alpha}$ for some $\alpha$. Hence, there exists $(p \approx q) \in T^{0}$, with

$$
V_{A}\left(T^{0}\right) \varsubsetneqq V_{A}\left(T^{0} \backslash p \approx q\right) .
$$

This shows that $T$ is $A$-independent and so the chain has upper bound. Therefore $\mathcal{F}$ has a maximal element $S^{\prime}$. Suppose $V_{A}(S) \varsubsetneqq V_{A}\left(S^{\prime}\right)$. So, there exists an element $\bar{a} \in V_{A}\left(S^{\prime}\right) \backslash V_{A}(S)$, and hence there is an equation $\left(p_{1} \approx q_{1}\right) \in S$, such that $p_{1}(\bar{a}) \neq q_{1}(\bar{a})$. Let $S^{\prime \prime}=S^{\prime}+\left(p_{1} \approx\right.$ $\left.q_{1}\right)$. We show that $S^{\prime \prime} \in \mathcal{F}$. Let $T_{0} \subseteq S^{\prime \prime}$ be finite. If $T_{0} \subseteq S^{\prime}$, then there is $(p \approx q) \in T_{0}$ with

$$
V_{A}\left(T_{0}\right) \varsubsetneqq V_{A}\left(T_{0} \backslash p \approx q\right) .
$$

If $\left(p_{1} \approx q_{1}\right) \in T_{0}$, then $T_{0} \backslash p_{1} \approx q_{1} \subseteq S^{\prime}$, and hence

$$
\bar{a} \in V_{A}\left(T_{0} \backslash p_{1} \approx q_{1}\right) .
$$

But, since $p_{1}(\bar{a}) \neq q_{1}(\bar{a})$, so $\bar{a}$ does not belong to $V_{A}\left(T_{0}\right)$. Therefore

$$
V_{A}\left(T_{0}\right) \varsubsetneqq V_{A}\left(T_{0} \backslash p_{1} \approx q_{1}\right),
$$

and hence $S^{\prime \prime} \in \mathcal{F}$, which is impossible. Hence we must have $V_{A}(S)=$ $V_{A}\left(S^{\prime}\right)$.

A topological space $M$ is $\omega$-cocompact if and only if for any countable open covering $M=\bigcup_{i=1}^{\infty} C_{i}$, there exists $m \geq 1$ such that for all $j_{1}, j_{2} \geq m$, we have

$$
\bigcup_{i=j_{1}}^{\infty} C_{i}=\bigcup_{i=j_{2}}^{\infty} C_{i}
$$


Lemma 2. If a topological space $M$ is Artinian, then every subset of $M$ is $\omega$-cocompact. The converse is also true.

Proof. We first show that for an Artinian topological space $M$, every $N \subseteq M$ is also Artinian. Suppose

$$
Y_{1}^{\prime} \subseteq Y_{2}^{\prime} \subseteq Y_{3}^{\prime} \subseteq \cdots
$$

is a chain of closed sets in $N$. We have $Y_{i}^{\prime}=Y_{i} \cap N$, for all $i$, where $Y_{i}$ is closed in $M$. Let $V_{i}=Y_{1} \cup \cdots \cup Y_{i}$. Then $V_{i}$ is closed in $M$ and we have

$$
V_{1} \subseteq V_{2} \subseteq V_{3} \subseteq \cdots .
$$

So, there is $m$ such that $V_{m}=V_{m+1}=V_{m+2}=\cdots$. This shows that

$$
Y_{1} \cup \cdots \cup Y_{m}=Y_{1} \cup \cdots \cup Y_{m} \cup Y_{m+1}=\cdots,
$$

and hence

$$
Y_{1}^{\prime} \cup \cdots \cup Y_{m}^{\prime}=Y_{1}^{\prime} \cup \cdots \cup Y_{m}^{\prime} \cup Y_{m+1}^{\prime}=\cdots,
$$

which implies that $Y_{m}^{\prime}=Y_{m+1}^{\prime}=\cdots$. This shows that $N$ is also Artinian. Now, suppose $M$ is Artinian and $M=\bigcup_{i=1}^{\infty} C_{i}$. We have $C_{i}=M \backslash D_{i}$, with $D_{i}$ closed. Then $\bigcap_{i=1}^{\infty} D_{i}=\emptyset$. Define

$$
Y_{m}=\bigcap_{i=m}^{\infty} D_{i} .
$$

Then $Y_{1} \subseteq Y_{2} \subseteq Y_{3} \subseteq \cdots$ is a chain of closed sets and so there exists $m$ with $Y_{m}=Y_{m+1}=\cdots$. Hence, for $j_{1}, j_{2} \geq m$ we have

$$
\bigcap_{i=j_{1}}^{\infty} D_{i}=\bigcap_{i=j_{2}}^{\infty} D_{i} \text {, }
$$

and so

$$
\bigcup_{i=j_{1}}^{\infty} C_{i}=\bigcup_{i=j_{2}}^{\infty} C_{i} .
$$

Hence $M$ is $\omega$-cocompact. Conversely, suppose $M$ is $\omega$-cocompact and $Y_{1} \subseteq Y_{2} \subseteq Y_{3} \subseteq \cdots$ is a chain of closed subsets of $M$. Then we have

$$
M=\bigcup_{i=1}^{\infty} Y_{i}^{c} \cup M,
$$

where the superscript ${ }^{c}$ denotes the complement. So, there exists $m$ such that for all $j_{1}, j_{2} \geq m$,

$$
\bigcup_{i=j_{1}}^{\infty} Y_{i}^{c}=\bigcup_{i=j_{2}}^{\infty} Y_{i}^{c} .
$$


This implies that

$$
\bigcap_{i=m}^{\infty} Y_{i}=\bigcap_{i=m+1}^{\infty} Y_{i}=\cdots
$$

Therefore, we have

$$
Y_{m}=\bigcap_{i=m+1}^{\infty} Y_{i}=\bigcap_{i=m+2}^{\infty} Y_{i}
$$

Similarly, we have

$$
Y_{m+1}=\bigcap_{i=m+2}^{\infty} Y_{i}=\cdots .
$$

Hence $Y_{m}=Y_{m+1}=\cdots$, and so $M$ is Artinian.

By this lemma, a domain $A$ is equational Artinian, if and only if, for all $n$, any subset of $A^{n}$ is $\omega$-cocompact. Let $A$ be an algebra and $S$ be a system of equation. We say that $S$ is $A$-stable, if for any proper finite subset $S^{\prime} \subseteq S$, we have $V_{A}(S)=V_{A}\left(S \backslash S^{\prime}\right)$.

Theorem 11. Let $A$ be an equational domain which is weak equational noetherian. Suppose for any system $S$, there exists a finite subset $S_{0}$ such that $S \backslash S_{0}$ is A-stable. Then $A$ is equational Artinian. The converse is true if in addition, the language $\mathcal{L}$ is countable.

Proof. Suppose for any system $S$, there exists a finite subset $S_{0}$ such that $S \backslash S_{0}$ is $A$-stable. We show that $A^{n}$ is $\omega$-cocompact. Let $A=$ $\bigcup_{i=1}^{\infty} C_{i}$ be an open covering. We have $C_{i}=A^{n} \backslash V_{A}\left(S_{i}\right)$ for some finite $S_{i}$. So, $\bigcap_{i=1}^{\infty} V_{A}\left(S_{i}\right)=\emptyset$ and hence $V_{A}\left(\bigcup_{i=1}^{\infty} S_{i}\right)=\emptyset$. Suppose $S=\bigcup_{i=1}^{\infty} S_{i}$. Then, there exists a finite subset $S_{0} \subseteq S$ such that $S \backslash S_{0}$ is $A$-stable. Since $S_{0}$ is finite, so there exists $m$ such that for all $j \geq m$, the set $V_{A}\left(\bigcup_{i=j}^{\infty} S_{i}\right)$ does not depend on $j$. Hence for all $j_{1}, j_{2} \geq m$, we have

$$
\bigcap_{i=j_{1}}^{\infty} V_{A}\left(S_{i}\right)=\bigcap_{i=j_{2}}^{\infty} V_{A}\left(S_{i}\right) .
$$

This is equivalent to

$$
\bigcup_{i=j_{1}}^{\infty} C_{i}=\bigcup_{i=j_{2}}^{\infty} C_{i}
$$

and hence $A^{n}$ is $\omega$-cocompcat. Now, suppose the language $\mathcal{L}$ is countable and $A$ is equational Artinian. Let $S$ be a system of equations. 
We index $S$ by natural numbers as $S=\left\{p_{i} \approx q_{i}\right\}_{i=1}^{\infty}$. Suppose $C=$ $A^{n} \backslash V_{A}(S)$. We have

$$
C=\bigcup_{i=1}^{\infty}\left(A^{n} \backslash V_{A}\left(p_{i} \approx q_{i}\right)\right)
$$

so by our assumption on $\omega$-cocompactness of $A$, there exists $m$ such that for all $j_{1}, j_{2} \geq m$,

$$
\bigcup_{i=j_{1}}^{\infty}\left(A^{n} \backslash V_{A}\left(p_{i} \approx q_{i}\right)\right)=\bigcup_{i=j_{2}}^{\infty}\left(A^{n} \backslash V_{A}\left(p_{i} \approx q_{i}\right)\right) .
$$

Let $S_{0}=\left\{p_{1} \approx q_{1}, \ldots, p_{m-1} \approx q_{m-1}\right\}$. Therefore for any $j$, we have

$$
V_{A}\left(S \backslash S_{0}\right)=V_{A}\left(S \backslash\left(S_{0}+p_{m} \approx q_{m}+\cdots+p_{m+j} \approx q_{m+j}\right)\right),
$$

so $S \backslash S_{0}$ is $A$-stable.

Acknowledgement The authors would like to thank Andreas Blass, Anton Klyachko, Gerhard Paseman and Benjamin Steinberg for their useful comments and suggestions during a discussion in MathOverflow.

\section{REFERENCES}

[1] Baumslag G., Myasnikov A., Remeslennikov V. Algebraic geometry over groups, I. Algebraic sets and ideal theory. J. Algebra, 1999, 219, pp. 16-79.

[2] Baumslag G., Myasnikov A., Remeslennikov V. Discriminating completions of hyperbolic groups. J. Group Theory, 2000, 3 (4), pp. 467-479.

[3] Baumslag G., Myasnikov A., Romankov V. Two theorems about equationally noetherian groups. J. Algebra, 1997, 194, pp. 654-664.

[4] Kharlampovich O., Myasnikov A. Irreducible affine varieties over a free group. I: irreducibility of quadratic equations and Nullstellensatz. J. Algebra, 1998, 200 (2) pp. 472-516.

[5] Myasnikov A., Remeslennikov V. Algebraic geometry over groups, II. Logical Fundations. J. Algebra, 2000, 234, pp. 225-276.

[6] Kharlampovich O., Myasnikov A. Tarski's problem about the elementary theory of free groups has a psitive solution. E.R.A. of AMS, 1998, 4 pp. 101-108.

[7] Sela Z. Diophantine geometry over groups:I-X. preprints, Arxiv.

[8] Kharlampovich O., Myasnikov A. The elemntary theory of free non-abelian groups. J. Algebra, 2006, 302, pp. 451-552.

[9] Daniyarova E., Remeslennikov V. Bounded algebraic geometry over free Lie algebras. Algebra and Logic, 2005, 44 (3), pp. 148-167.

[10] Morar P., Shevlyakov A. Algebraic geometry over additive positive monoids: Systems of coefficient free equations. Combinatorial and Geometric group Theory: Dortmund and Carleton Conferences, 2010, pp. 261-278.

[11] Shevlyakov A. Algebraic geometry over additive monoid of natural numbers: the classification of coordiante monoids. Groups, Complexity and Cryptology, 2010, 2 (1), pp. 91-111.

[12] Daniyarova E., Myasnikov A., Remeslennikov V. Unification theorems in algebraic geometry. Algebra and Discrete Mathamatics, 2008, 1, pp. 80-112. 
[13] Daniyarova E., Myasnikov A., Remeslennikov V. Algebraic geometry over algebraic structures, II: Fundations. J. Math. Sci., 2012, 185 (3), pp. 389-416.

[14] Daniyarova E., Myasnikov A., Remeslennikov V. Algebraic geometry over algebraic structures, III: Equationally noetherian property and compactness. South. Asian Bull. Math., 2011, 35 (1), pp. 35-68.

[15] Daniyarova E., Myasnikov A., Remeslennikov V. Algebraic geometry over algebraic structures, IV: Equatinal domains and co-domains. Algebra and Logic, 49 (6), pp. 483-508.

[16] Burris S., Sankappanavar, H.P. A course in universal algebra. Springer-Verlag, 1981.

[17] Gorbunov V.A., Algebraic theory of quasi-varieties. Perseus Books, 1998.

[18] Malcev A.I., Algebraic structures. Moscow, Nauka, 1987.

[19] Adian S. Infinite irreducible systems of group identities. Izv. Akad. Nauk SSSR Ser. Mat., 1970, 34, pp. 715-734.

[20] Shahryari M. Equational noetherian algebras and chain conditions. J. Siberian Federal University: Mathematics and Physics, 2013, 6 (4), pp. 521-526.

P. Modabberi: Department of Pure Mathematics, Faculty of Mathematical Sciences, University of TABriz, TABriz, Iran

E-mail address: p_modabberi@tabrizu.ac.ir

M. Shahryari: Department of Pure Mathematics, Faculty of Mathematical Sciences, University of TABriz, TABriz, Iran

E-mail address: mshahryari@tabrizu.ac.ir 